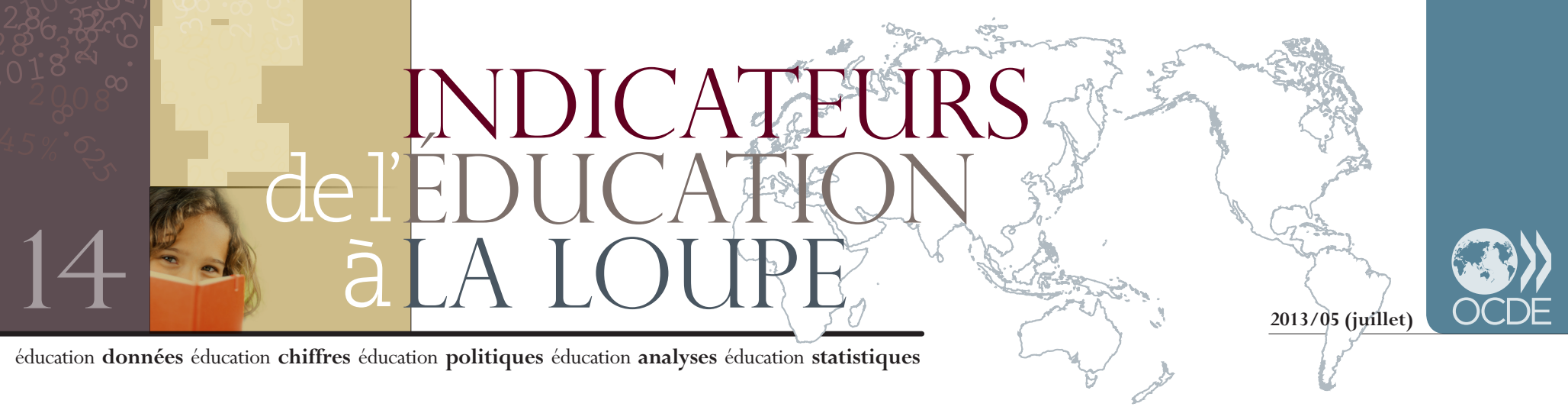

\title{
Quels facteurs influencent la mobilité internationale des étudiants ?
}

- Entre 2000 et 2011, le nombre d'étudiants en mobilité internationale a plus que doublé. Aujourd'hui, près de $\mathbf{4 . 5}$ millions d'étudiants suivent une formation de niveau tertiaire dans un pays dont ils ne sont pas ressortissants.

- Les effectifs les plus importants d'étudiants en mobilité internationale sont originaires de Chine, de Corée et d'Inde. Les étudiants asiatiques représentent $53 \%$ de l'effectif total d'étudiants en formation à l'étranger.

- Ces dernières décennies, le marché international de l'éducation a vu l'émergence de nouveaux acteurs, tels que I'Australie, I'Espagne, la Fédération de Russie, la Nouvelle-Zélande et, plus récemment, la Corée. À l'inverse, le pourcentage d'étudiants en mobilité internationale a reculé dans certains des pays d'accueil traditionnellement les plus attractifs - en Allemagne et aux États-Unis, par exemple.

- Les pays tirant un bénéfice croissant de la mobilité internationale des étudiants, la concurrence qu'ils se livrent pour attirer et retenir ces étudiants a entraîné ces dix dernières années un remaniement de la carte des pays d'accueil.

L'internationalisation de l'enseignement tertiaire est allée de pair avec son expansion.

Au cours des dernières décennies, le nombre d'étudiants suivant une formation tertiaire dans un pays dont ils ne sont pas ressortissants a connu une forte augmentation, reflétant l'expansion des systèmes d'enseignement tertiaire dans le monde et la mondialisation des économies et des sociétés. La plupart des étudiants qui suivent une formation à l'étranger choisissant un pays de I'OCDE comme pays d'accueil, l'expansion de l'enseignement tertiaire en dehors de la zone OCDE est venue alimenter les effectifs d'étudiants étrangers de cette dernière.

Le nombre d'étudiants suivant une formation tertiaire dans un pays dont ils ne sont pas ressortissants a plus que triplé, passant de 1.3 million en 1990 à près de 4.3 millions en 2011, soit un taux de croissance annuel moyen de près de $6 \%$ - un rythme plus soutenu que l'augmentation globale des effectifs de l'enseignement tertiaire dans le monde. Contrairement aux prévisions de certains observateurs, le taux de croissance de la mobilité internationale des étudiants n'a pas connu de recul durant la crise économique mondiale (voir le graphique page 2).

Parmi les bénéfices qu'un nombre croissant d'étudiants associent à des études à l'étranger figurent l'enrichissement culturel et l'amélioration des compétences linguistiques, l'obtention de diplômes prestigieux et un avantage compétitif en termes de débouchés professionnels. En suivant une formation à l'étranger, les étudiants ont la possibilité d'approfondir leur connaissance d'autres sociétés, langues, cultures et pratiques commerciales, et d'améliorer leurs perspectives sur le marché du travail (OCDE, 2004). En outre, la baisse des prix des transports internationaux et des télécommunications facilite également le départ des étudiants à l'étranger.

\section{Étudiants en mobilité internationale : qui va où?}

Selon les chiffres de 2011, les effectifs les plus importants d'étudiants en mobilité internationale viennent de Chine, de Corée et $d^{\prime}$ Inde. Les étudiants asiatiques représentent $53 \%$ de l'effectif total d'étudiants suivant une formation à l'étranger et parmi eux, 3 sur 4 choisissent d'étudier dans un pays de I'OCDE (voir la carte). Dans certains pays comme la Chine, les étudiants sont encouragés à suivre une formation à l'étranger dans une optique de renforcement des capacités (OCDE/ Banque mondiale, 2007).

Par étudiants en mobilité internationale, on entend les étudiants qui ont quitté leur pays d'origine pour se rendre dans un autre pays avec l'intention d'y suivre des études. L'Institut de statistique de I'UNESCO, I'OCDE et Eurostat définissent les étudiants en mobilité internationale comme ceux qui suivent une formation dans un autre pays que celui dont ils sont résidents ou dans lequel ils étaient scolarisés auparavant. En cas d'absence de données sur les étudiants en mobilité internationale, on utilise le concept d'étudiants étrangers.

Par étudiant étranger, on entend tout étudiant qui n'est pas ressortissant du pays où il suit une formation. Les étudiants en mobilité internationale constituent donc un sous-ensemble des étudiants étrangers. 
Les étudiants asiatiques sont suivis par les étudiants européens ( $23 \%$ ), notamment les ressortissants de pays membres de I'Union européenne (UE) également membres de I'OCDE (UE21) (14\%). Les étudiants africains représentent à eux seuls $12 \%$ de l'effectif mondial d'étudiants en mobilité internationale, tandis que les étudiants originaires du reste du monde n'en représentent que $12 \%$.

D'où viennent les étudiants étrangers ? Les pays d'origine les plus importants en 2011, par régions du monde
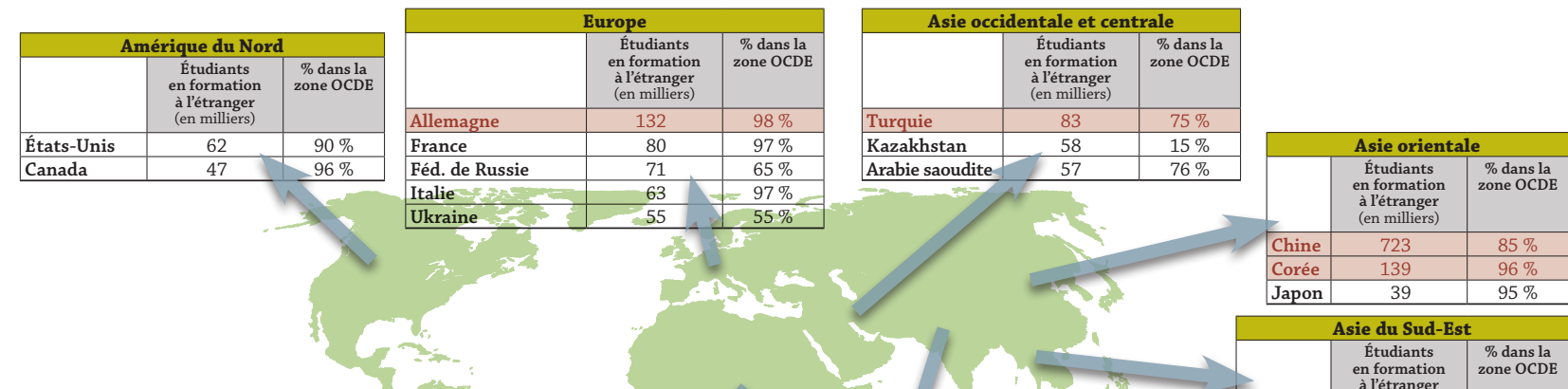

Ukraine

\begin{tabular}{|l|c|c|}
\hline \multicolumn{3}{|c|}{ Amérique latine et Caraïbes } \\
\hline & $\begin{array}{c}\text { Étudiants } \\
\text { en formation } \\
\text { à létranger } \\
\text { (en milliers) }\end{array}$ & $\begin{array}{c}\text { \% dans la } \\
\text { zone OCDE }\end{array}$ \\
\hline Brésil & 35 & $89 \%$ \\
\hline Colombie & 32 & $93 \%$ \\
\hline Mexique & 29 & $92 \%$ \\
\hline Pérou & 24 & $80 \%$ \\
\hline Venezuela & 19 & $69 \%$ \\
\hline
\end{tabular}
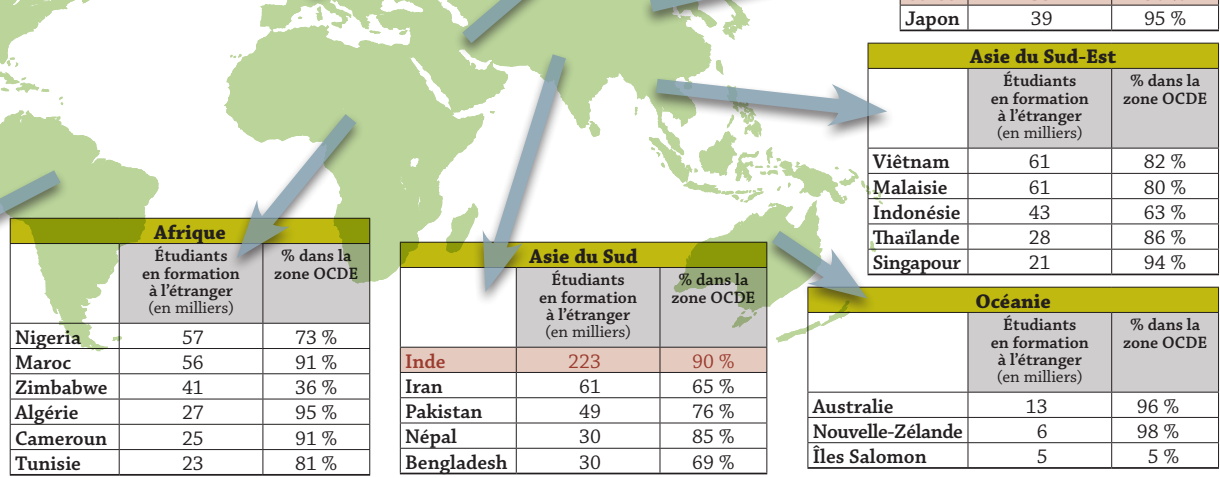

Remarque : les pays en rouge sont les pays d'où viennent le plus d'étudiants étrangers dans le monde. Source : OCDE et Institut de statistique de l'UNESCO

La mobilité internationale des étudiants reflète dans une large mesure les tendances migratoires inter- et intra-régionales. En outre, les flux d'étudiants à destination de pays d'Europe, d'Asie orientale et d'Océanie reflètent en général l'évolution géopolitique des régions, par exemple le resserrement des liens entre les pays de la région Asie-pacifique et le renforcement de la coopération au sein des pays européens, au-delà de I'Union européenne (UNESCO, 2009).

\section{La mondialisation : un vecteur de choix et de concurrence accrus pour les étudiants.}

Selon les chiffres de 2011, les pays de I'OCDE accueillent quelque $77 \%$ de l'effectif total d'étudiants suivant une formation dans un pays dont ils ne sont pas ressortissants. Au sein de la zone OCDE, près de la moitié de l'effectif total d'étudiants étrangers suit une formation dans l'un des cinq pays en tête du classement des pays d'accueil les plus prisés pour les formations tertiaires à l'étranger : les États-Unis, avec $17 \%$ de l'effectif mondial d'étudiants étrangers, suivis du Royaume-Uni (13\%), de l'Australie (6\%), de l'Allemagne (6 \%) et de la France (6\%).

Les étudiants en mobilité internationale privilégient les pays anglophones, où ils choisissent souvent de suivre une formation en sciences sociales, en commerce ou en droit (plus de $30 \%$ de l'effectif total suivent une formation dans ces domaines d'études). L'Australie, les États-Unis et le Royaume-Uni accueillent à eux seuls $36 \%$ de l'effectif mondial d'étudiants suivant une formation tertiaire à l'étranger, un pourcentage resté relativement stable depuis 2000, où il s'élevait à $39 \%$.

Les étudiants en mobilité internationale sont désormais plus susceptibles que par le passé de suivre les plus hauts niveaux de formation, illustrant l'internationalisation croissante de la recherche universitaire et des sciences (OCDE, 2009). En moyenne, dans les pays de l'OCDE, selon les chiffres de 2011, un étudiant suivant un programme de recherche de haut niveau sur cinq est un étudiant en mobilité internationale. Ce pourcentage passe la barre des $30 \%$ en Australie, en France, en Nouvelle-Zélande, aux Pays-Bas, au Royaume-Uni et en Suisse.

Ces dernières années, le marché international de l'éducation a vu l'émergence de nouveaux acteurs. Ainsi, selon les chiffres de 2011, un nombre important d'étudiants étrangers suivent une formation au Canada (5\%), en Espagne (2\%), en Fédération de Russie (4\%) et au Japon (4\%). À l'inverse, certains des pays d'accueil traditionnellement les plus prisés ont vu leur part de marché diminuer : entre 2000 et 2011, le pourcentage d'étudiants en mobilité internationale est ainsi passé de $23 \%$ à $17 \%$ aux États-Unis, tandis qu'en Allemagne, il a reculé de 3 points de pourcentage (voir le graphique ci-dessus). Bien que la Corée ne représente toujours qu'une petite part du marché international de l'éducation, elle a vu son nombre d'étudiants étrangers multiplié par plus de 17 au cours de cette même période. 
Évolution des parts sur le marché international de l'éducation $(2000,2011)$ Pourcentage de l'effectif total d'étudiants suivant une formation tertiaire à l'étranger, par pays d'accueil

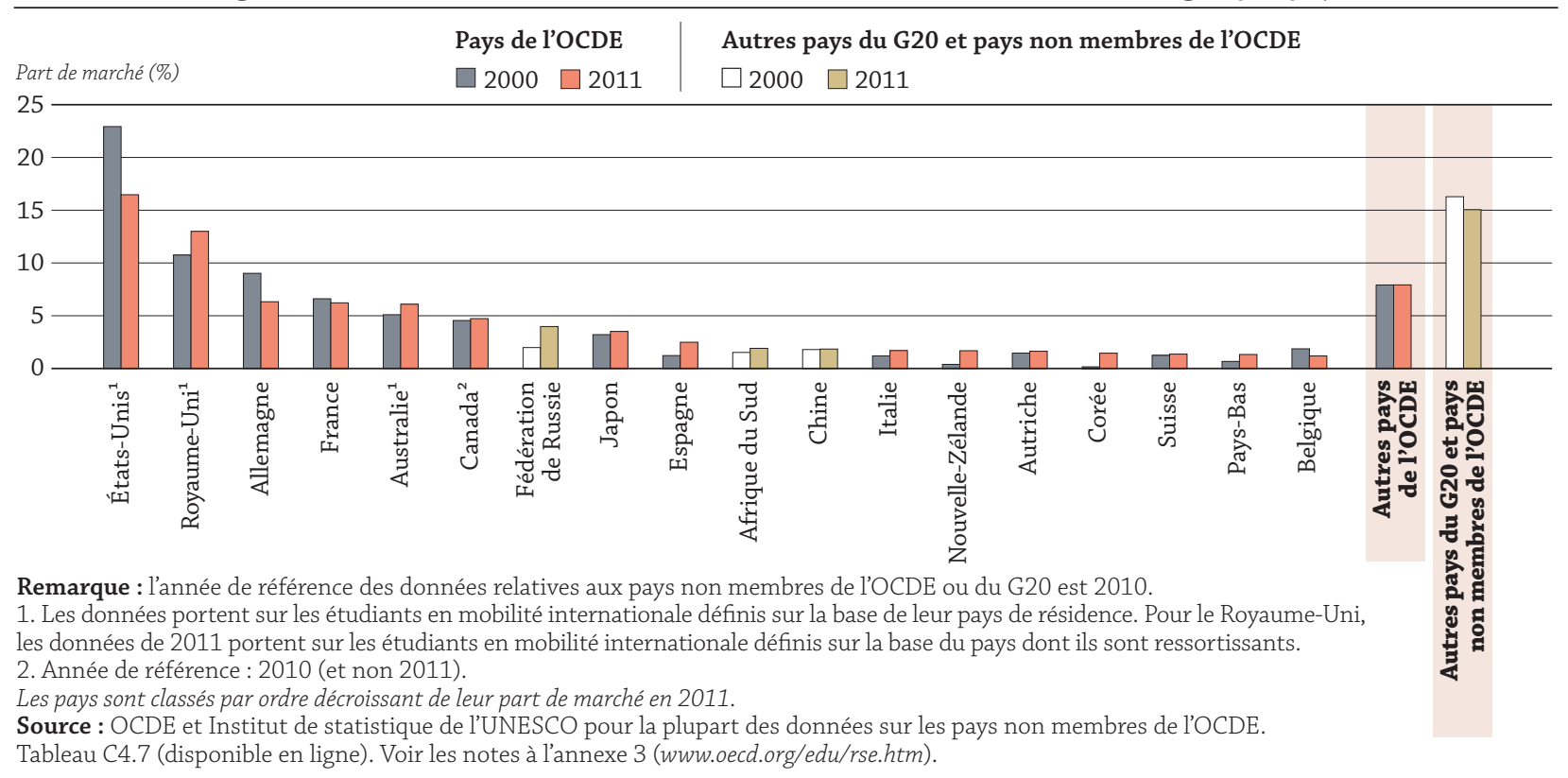

\section{Les frais de scolarité, la langue et les politiques d'immigration : autant de facteurs qui peuvent attirer les étudiants, ou les repousser...}

Le prestige du système d'enseignement supérieur d'un pays et de ses formations peut influer sur le pays d'accueil que l'étudiant choisira pour suivre une formation à l'étranger. Toutefois, les politiques d'immigration et la langue peuvent également constituer une passerelle ou un obstacle pour les étudiants en mobilité internationale. Les pays d'accueil anglophones s'avérant les plus attractifs en valeur absolue, un nombre croissant de pays non anglophones ont commencé à proposer des formations en anglais afin de pallier leur désavantage linguistique. Les politiques d'immigration ont aussi été récemment modifiées dans certains pays de l'OCDE : la Finlande et la Norvège ont ainsi amendé leur législation relative à la naturalisation afin de prendre en compte les années de résidence en qualité d'étudiant lors de l'évaluation de l'éligibilité à la citoyenneté, tandis que le Canada facilite l'octroi de la résidence permanente pour les diplômés en mobilité internationale (OCDE, 2012). À l'inverse, certains pays comme les États-Unis et, plus récemment, le Royaume-Uni, rendent plus difficile l'entrée des étudiants en mobilité internationale sur leur territoire.

Le montant des frais de scolarité demandés aux étudiants en mobilité internationale peut également influer sur l'attrait d'une destination. Les fournisseurs de services d'éducation, publics comme privés, voient de plus en plus dans les étudiants en mobilité internationale une source de revenus supplémentaires et tendent à augmenter les frais de scolarité de ces derniers. Dans la majorité des pays de l'OCDE dont les données sont disponibles, les frais de scolarité demandés par les établissements d'enseignement public sont plus élevés pour les étudiants en mobilité internationale que pour les ressortissants nationaux suivant la même formation (voir le tableau ci-dessous).

\section{Structure des frais de scolarité dans les pays de l'OCDE}

\section{Structure des frais de scolarité}

Frais de scolarité plus élevés pour les étudiants en mobilité internationale que pour les ressortissants nationaux

\section{Pays de l'OCDE}

Australie, Autriche ${ }^{1}$, Belgique $^{1}$, Canada, Danemark ${ }^{1}$, Estonie $^{1}$, États-Unis, Fédération de Russie, Irlande, Islande ${ }^{1}$,

Nouvelle-Zélande, Pays-Bas ${ }^{1}$, Pologne ${ }^{1}$, République tchèque ${ }^{1}$, Royaume-Uni ${ }^{1}$, Suède ${ }^{1}$ et Turquie

Frais de scolarité identiques pour les étudiants en mobilité $\quad$ Allemagne, Corée, Espagne, France, Italie, Japon, Mexique internationale et les ressortissants nationaux

Pas de frais de scolarité, ni pour les étudiants en mobilité internationale ni pour les ressortissants nationaux

et Suisse

Finlande et Norvège

1. Pour les étudiants originaires de pays tiers de l’Union européenne et de l'Espace économique européen. 
... pour autant, des frais de scolarité élevés ne sont pas forcément synonymes de faible àttractivité pour un pays.

Dans certains pays comme l'Australie, les États-Unis ou le Royaume-Uni, le niveau élevé des frais de scolarité ne constitue pas nécessairement un obstacle pour les étudiants en mobilité internationale. Dans certains cas, ces derniers peuvent bénéficier de prêts ou de bourses pour poursuivre leurs études dans ces pays; dans d'autres, les formations restent attractives en raison des débouchés professionnels qu'elles offrent dans le pays d'accueil (comme en Australie et en Nouvelle-Zélande). Toutefois, le recul de la part de marché des États-Unis peut s'expliquer en partie par le niveau comparativement élevé des frais de scolarité qui y sont demandés aux étudiants en mobilité internationale, notamment dans un contexte de rude concurrence de la part d'autres pays d'accueil, principalement anglophones, offrant des possibilités de formation similaires à un moindre coût.

D'un autre côté, les pays nordiques affichent un pourcentage d'étudiants étrangers moins élevé par rapport à tous les autres pays, alors qu'ils proposent certaines formations en anglais et appliquent des frais de scolarité faibles, voire nuls, pour les étudiants, qu'ils soient ressortissants nationaux ou étrangers. Par ailleurs, en Suède, après l'introduction de frais de scolarité pour les étudiants étrangers, le nombre de ceux ne venant pas de pays membres de l'EEE ou de Suisse a reculé de près de $60 \%$, passant de 10234 au 4 e trimestre 2010 à 4269 au 4 e trimestre 2011.

\section{Les étudiants étrangers représentent une manne pour les pays d'accueil, tant à court qu'à long terme.}

Les fournisseurs publics et privés d'éducation voient également de plus en plus dans l'exportation de leurs services d'enseignement supérieur aux étudiants en mobilité internationale une source de revenus supplémentaires et tendent à augmenter leurs tarifs pour ces derniers. En plus des frais de scolarité versés aux établissements d'enseignement publics et privés, les recettes générées par les étudiants en mobilité internationale pendant leurs études peuvent être substantielles, notamment en termes de frais de subsistance (Commission européenne, 2013). Au Canada, les dépenses consenties par les étudiants en mobilité internationale au titre de leurs frais de scolarité ainsi que de leurs frais de logement et de subsistance ont contribué à hauteur de plus de 8 milliards CAD à l'économie en 2010. Ce montant est supérieur « à la valeur totale des exportations canadiennes d'aluminium à l'état brut (6 milliards CAD), d'hélicoptères, d'avions et d'astronefs (6.9 milliards CAD) » (Canada, 2012).

Lorsque les étudiants étrangers font le choix de s'installer dans leur pays d'accueil après l'obtention de leur diplôme, ils ont un impact à long terme sur l'économie. Dans l'ensemble, dans les pays de l'OCDE dont les données de 2008 et 2009 sont disponibles, le taux d'installation atteint $25 \%$ et s'établit à plus de $20 \%$ dans la grande majorité d'entre eux. En Australie, au Canada, en France et en République tchèque, ce taux passe même la barre des $30 \%$, ce qui n'est pas sans influer sur le marché du travail.

\section{Références}

Canada (2012), L'éducation internationale : Un moteur-clé de la prospérité future du Canada, Rapport final du comité consultatif sur la Stratégie du Canada en matière d'éducation internationale, Canada.

Commission européenne (2013), Immigration of International Students to the EU, European Migration Network Study 2012, Commission européenne.

OCDE (2004), Enseignement supérieur : Internationalisation et commerce, Éditions OCDE.

OCDE (2009), L'enseignement supérieur à l'horizon 2030, Volume 2 : Mondialisation, Éditions OCDE.

OCDE (2012), Perspectives des migrations internationales 2012, Éditions OCDE.

OCDE/Banque mondiale (2007), L'enseignement supérieur transnational : Un levier pour le développement, Éditions OCDE.

UNESCO (2009), Recueil de données mondiales sur l'éducation, Institut de statistique de I'UNESCO, Montréal.

Pour conclure Le nombre d'étudiants suivant une formation dans un pays dont ils ne sont pas ressortissants a augmenté de façon spectaculaire au cours des dernières décennies, les étudiants asiatiques représentant en $201153 \%$ de l'effectif total d'étudiants en formation à l'étranger. Le marché international de l'éducation voit l'émergence de nouveaux acteurs en raison de la mise en ouvre de stratégies plus vastes pour attirer et retenir les migrants hautement qualifiés, source de revenus à court et à long terme pour les pays.

\begin{tabular}{l|l}
$\begin{array}{l}\text { Voir : } \\
\text { www.oecd.org/ }\end{array}$ & Consulter : \\
edu/rse.htm & OCDE (2013), Regards sur l'éducation 2013: \\
& Les indicateurs de l'OCDE, Éditions OCDE. \\
&
\end{tabular}

\section{Pour plus d'informations,} contacter:

Cuauhtemoc Rebolledo-Gómez (Cuauhtemoc.Rebolledo-Gomez@oecd.org) Joris Ranchin (Joris.Ranchin@oecd.org)
Prochain numéro :

Combien d'étudiants entreront à l'université ? Et combien en sortiront diplômés? 